\title{
Quantum properties of the codirectional three-mode Kerr nonlinear coupler
}

\author{
Faisal A. A. El-Orany, ${ }^{1}$ M. Sebawe Abdalla, ${ }^{2}$ and and J. Peřina ${ }^{3}$ \\ 1 Department of Mathematics and Computer Science, \\ Faculty of Science, Suez Canal University, Ismailia, \\ Egypt; Department of Optics, Palacký University, \\ 17. listopadu 50, 772 07 Olomouc, Czech Republic \\ ${ }^{2}$ Mathematics Department, College of Science, \\ King Saud University, P.O. Box 2455, Riyadh 11451, Saudi Arabia \\ ${ }^{3}$ Department of Optics and Joint Laboratory of Optics, \\ Palacký University, 17. listopadu 50, 77207 Olomouc, Czech Republic
}

(Dated: October 23, 2018)

\begin{abstract}
We investigate the quantum properties for the codirectional three-mode Kerr nonlinear coupler. We investigate single-, two- and three-mode quadrature squeezing, Wigner function and purity. We prove that this device can provide richer nonclassical effects than those produced by the conventional coupler, i.e. the two-mode Kerr coupler. We show that it can provide squeezing and the quadrature squeezing exhibiting leaf-revival-collapse phenomenon in dependence on the values of the interaction parameters. In contrast to the conventional Kerr coupler two different forms of cat states can be simultaneously generated in the waveguides. We deduce conditions required for the complete disentanglement between the components of the system.

PACS numbers: 42.50Dv,42.60.Gd
\end{abstract}

Keywords: Quasiprobability functions; nonlinear coupler; squeezed light; quantum phase

\section{INTRODUCTION}

The optical coupler is a device composed of two (or more) waveguides, which are placed close enough to allow exchanging energy between waveguides via evanescent waves [1]. Re- 
cently, this device has attracted much attention for several reasons. The progress in the optics communication and quantum computing networks requires data transmission [2]. This simple device has potential applications in all-optical switching [3, 4]. Furthermore, it provides electromagnetic fields with an exceptionally wide range of nonclassical effects. Most importantly this device has been implemented [5, 6] and applied in many experimental approaches, e.g. in picosecond switching induced by saturable absorption [7], optical multi-mode interference devices based on self-imaging [8] and photonic bandgap structures in planar nonlinear waveguides [9]. Also the generation of correlated photons in controlled spatial modes by downconversion in nonlinear waveguides has been discussed in [10].

Quantum mechanically, there are different types of directional couplers. For instance, symmetric coupler [11] (linear (or nonlinear) processes are involved in both the waveguides), asymmetric coupler [12] (at least one of the waveguides possesses different nonlinearity than the others), Raman-Brillouin coupler [13], bandgap coupler [14] and Kerr coupler [15, 16, 17, 18, 19, 20, 21, 22]. For more details about their properties the reader can consult the review papers [23].

Kerr nonlinear coupler (KNC) has taken a considerable interest in the literature since the third-order nonlinearity provides an effective mechanism for generating nonclassical effects in the electromagnetic waves caused by the processes of self-phase and cross-phase modulations. Also this is related to potential advantage of the possible observation of the large values of third-order optical nonlinearities in the organic polymers [5]. In this regard the generation of continous variable Einstein-Podolsky-Rosen entanglement via Kerr nonlinearity in an optical fiber [24] as well as the generation of spatial soliton arrays in a planar Kerr waveguide from seeded spontaneous parametric down conversion [25] are achieved. Several papers have been devoted to the quantum properties of the $\mathrm{KNC}$ in the framework of rotating-wave approximation by neglecting the rotational terms in such a way that a closed form solution is obtained [15, 16, 17, 18, 19, 20]. In this case $\mathrm{KNC}$ has provided many of interesting effects such as revival-collapse phenomenon (RCP) in the mean-photon numbers, squeezing of vacuum fluctuations, sub-Poissonian statistics in single as well as in the compound modes [15]. The phase distribution of KNC has been investigated showing that the phase-difference evolution is closely connected with the energy exchange between waveguides and the RCP in the mean-photon numbers is due to the bifurcation of the phase-difference probability distribution [17]. Furthermore, the geometry of the waveguides has been considered via 
varying linear coupling coefficients in the codirectional KNC [16] and contradirectional KNC $[19,20]$. In these cases it has been shown that there is a possibility to control the switching characteristics and principal squeezing effect by adjusting the shape of the waveguides. Quite recently, we have investigated the single-mode quantum properties of the codirectional Kerr nonlinear coupler when the frequency mismatch is involved and a condition for obtaining an exact solution for the equations of motion is fulfilled [21, 22]. For this case we have shown that the mean-photon numbers exhibit oscillatory behaviour rather than revivals and collapses. Additionally we have proved that the Schrödinger-cat states, in particular, YurkeStoler cat states (YSCS) [26] can be generated. Also the higher-order squeezing has been investigated [22].

Till now the KNC has been treated as a two-mode device. In the present paper we give for the first time-as far as we know-the three-mode version of this device. The motivation of developing such device is that the three-mode $\mathrm{KNC}$ can provide nonclassical effects richer than those obtained from the conventional coupler (i.e. the two-mode version), as we shall show throughout the paper. As is well known that the basic efforts in the quantum optics is to enhance the nonclassical effects. Additionally, we show that the quadrature squeezing exhibits leaf-revival-collapse phenomenon in dependence on the values of the interaction parameters. In this phenomenon the revival patterns provide leaf shapes and between two revival patterns short collapse period occurs. It is worth reminding that for the standard revival-collapse phenomenon the revival patterns have ellipsoid shape. We proceed that, in contrast to the conventional Kerr coupler two different forms of cat states can be simultaneously generated in the waveguides. The investigation of the three-mode $\mathrm{KNC}$ will be given in the following order: In section 2 we give the Hamiltonian for the system and the solution for the equations of motion. Also we derive the expectation values for different moments of operators, which will be used in the paper. In section 3 we investigate the quadrature squeezing. In section 4 we discuss the evolution of the Wigner function and the purity for the single-mode case. In section 5 we give the main conclusions from the results.

\section{MODEL FORMALISM AND DYNAMICAL SOLUTION}

In this section we give the Hamiltonian for the system and derive the solutions for its equations of motion. 
The Hamiltonian controlling the three-mode codirectional Kerr nonlinear coupler can be represented as

$$
\begin{aligned}
& \frac{\hat{H}}{\hbar}=\sum_{j=1}^{3}\left(\omega_{j} \hat{a}_{j}^{\dagger} \hat{a}_{j}+\chi_{j} \hat{a}_{j}^{\dagger 2} \hat{a}_{j}^{2}\right)+\bar{\chi}_{1} \hat{a}_{1}^{\dagger} \hat{a}_{1} \hat{a}_{2}^{\dagger} \hat{a}_{2}+\bar{\chi}_{2} \hat{a}_{1}^{\dagger} \hat{a}_{1} \hat{a}_{3}^{\dagger} \hat{a}_{3}+\bar{\chi}_{3} \hat{a}_{2}^{\dagger} \hat{a}_{2} \hat{a}_{3}^{\dagger} \hat{a}_{3} \\
& +\lambda_{1}\left[\hat{a}_{1} \hat{a}_{2}^{\dagger} \exp \left(i \Delta_{1} t\right)+\hat{a}_{1}^{\dagger} \hat{a}_{2} \exp \left(-i \Delta_{1} t\right)\right]+\lambda_{2}\left[\hat{a}_{1} \hat{a}_{3}^{\dagger} \exp \left(i \Delta_{2} t\right)+\hat{a}_{1}^{\dagger} \hat{a}_{3} \exp \left(-i \Delta_{2} t\right)\right],
\end{aligned}
$$

where $\Delta_{1}=\omega_{1}-\omega_{2}$, and $\Delta_{2}=\omega_{1}-\omega_{3}$. The waves are designated by $\hat{a}_{1}$ (fundamental and/or first), $\hat{a}_{2}$ (second) and $\hat{a}_{3}$ (third) modes with frequencies $\omega_{1}, \omega_{2}$ and $\omega_{3}$, respectively. The coupling constants $\chi_{j}$ and $\bar{\chi}_{j}$ are proportional to the third-order susceptibility $\chi^{(3)}$ and are responsible correspondingly for the self-action and cross-action processes of the $j$ th mode. The linear coupling between the waveguides are represented by $\lambda_{1}$ and $\lambda_{2}$, which we assume to be real. Hamiltonian (11) gives a generalization to several models discussed in the literatures earlier by controlling the values of the interaction parameters such as anhramonic oscillator [27], up-conversion process [28] and two-mode KNC [21]. The scheme describing Hamiltonian (11) is shown in Fig. 1 and can be explained as follows. Two waveguides are operating by Kerr-like nonlinear processes. In the first waveguide the fundamental mode $\hat{a}_{1}$ propagates, while in the second waveguide the second $\hat{a}_{2}$ and third $\hat{a}_{3}$ modes propagate. The interaction between the fundamental mode and the second-third modes occurs via the evanescent waves. We have assumed that the all waves are propagating with the same velocity $v$, hence the time $t$ and travelled distance $z$ are related by $z=v t$. Outgoing fields can be detected as single or compound modes by means of homodyne, photocounting or coincidence detection in the standard way. Throughout the investigation of the system we do not consider dissipation, which generally decreases the amount of nonclassical effects.

We have to comment that the linear coupling coefficient is from the wave equation equal to $\lambda / v=\omega^{2} \chi^{(2)} / 2 k c^{2}$, whereas the nonlinear coupling coefficients are $\chi / v=\omega^{2} \chi^{(3)} / 2 k c^{2}(v$ being the speed of light in the medium, $\omega$ being frequency of the travelled wave and $k$ is its wave number). Taking into account that values of quadratic susceptibilities lie in an interval $10^{-14}-10^{-10} \mathrm{~m} / \mathrm{V}$ and values of cubic susceptibilities in an interval $10^{-17}-10^{-13} \mathrm{~m}^{2} / V^{2}$ for various nonlinear materials, then in the optical region the values of $\lambda / v$ lie from $10^{4}$ to $10^{7} \mathrm{~m}^{-1}$ and values of $\chi / v$ from 10 to $10^{5} \mathrm{~m}^{-1}$. So the effects predicted should be expected to be observable in samples of the length of centimeters and in nanosecond scales.

Now introducing new operators $\hat{A}_{j}=\hat{a}_{j} \exp \left(i \omega_{j} t\right), j=1,2,3$ we can write the Heisenberg 


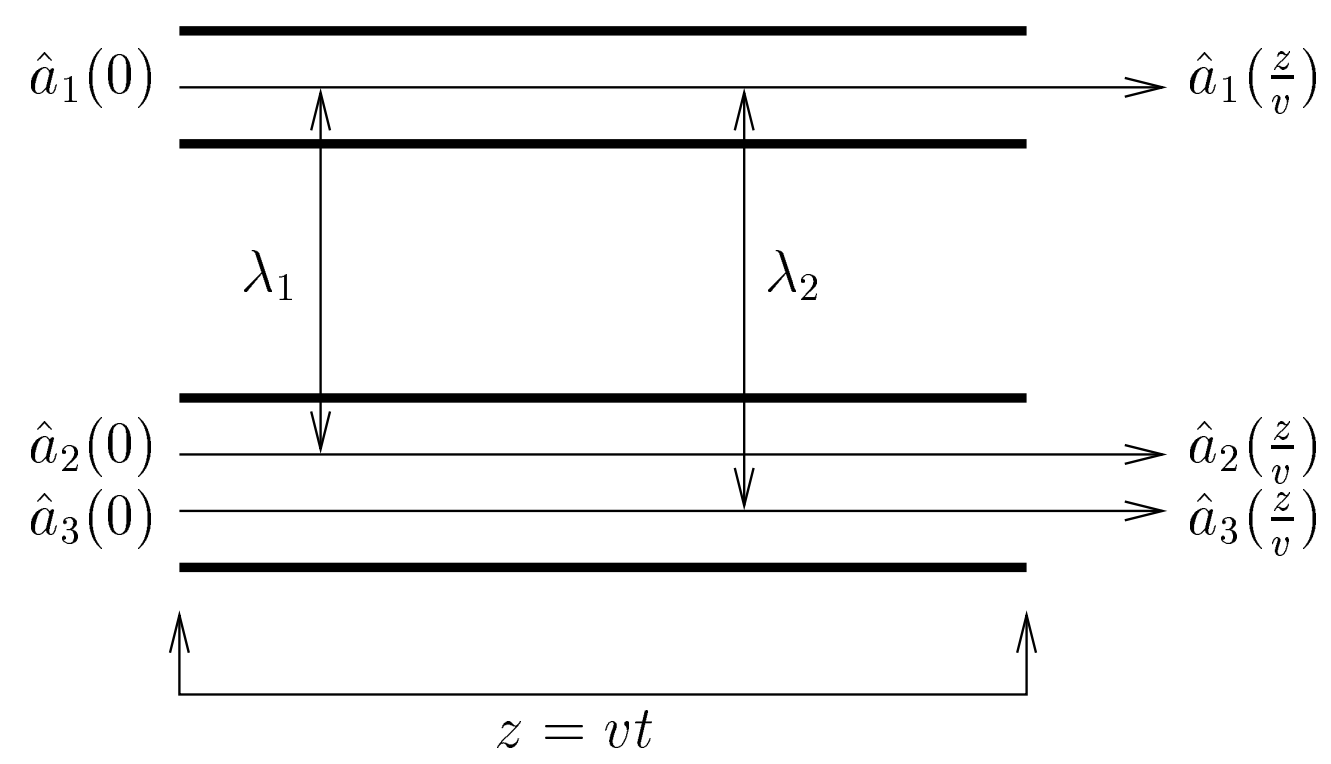

FIG. 1: Scheme of realization of interaction (1).

equations for (11) as

$$
\begin{aligned}
& \frac{d \hat{A}_{1}}{d t}=-i\left(2 \chi_{1} \hat{A}_{1}^{\dagger} \hat{A}_{1}+\bar{\chi}_{1} \hat{A}_{2}^{\dagger} \hat{A}_{2}+\bar{\chi}_{2} \hat{A}_{3}^{\dagger} \hat{A}_{3}\right) \hat{A}_{1}-i \lambda_{1} \hat{A}_{2}-i \lambda_{2} \hat{A}_{3}, \\
& \frac{d \hat{A}_{2}}{d t}=-i\left(2 \chi_{2} \hat{A}_{2}^{\dagger} \hat{A}_{2}+\bar{\chi}_{1} \hat{A}_{1}^{\dagger} \hat{A}_{1}+\bar{\chi}_{3} \hat{A}_{3}^{\dagger} \hat{A}_{3}\right) \hat{A}_{2}-i \lambda_{1} \hat{A}_{1}, \\
& \frac{d \hat{A}_{3}}{d t}=-i\left(2 \chi_{3} \hat{A}_{3}^{\dagger} \hat{A}_{3}+\bar{\chi}_{2} \hat{A}_{1}^{\dagger} \hat{A}_{1}+\bar{\chi}_{3} \hat{A}_{2}^{\dagger} \hat{A}_{2}\right) \hat{A}_{3}-i \lambda_{2} \hat{A}_{1} .
\end{aligned}
$$

To solve (2) exactly we assume the processes of the self-action and cross-action compensate each other in the evolution of the system. This can be expressed as $\chi=\chi_{1}=\chi_{2}=\chi_{3}, \bar{\chi}=$ $\bar{\chi}_{1}=\bar{\chi}_{2}=\bar{\chi}_{3}$ and $2 \chi=\bar{\chi}$. In fact, the cross-spectral coupling between various waveguides may be comparable with the self-coupling provided that the surfaces of the waveguides are of high quality (roughness is much less than the wavelength) to avoid a substantional reduction of amplitudes by evanescent damping. As follows from the values for quadratic and cubic susceptibilites both the linear and nonlinear couplings may be simultaneously significant for sufficiently strong fields. Under these conditions one can easily prove that

$$
\hat{A}_{1}^{\dagger} \hat{A}_{1}+\hat{A}_{2}^{\dagger} \hat{A}_{2}+\hat{A}_{3}^{\dagger} \hat{A}_{3}=\hat{N}
$$

is a constant of motion. Based on this fact the system of equations (2) can be modified via the substitution $\hat{B}_{j}=\exp (2 i \chi \hat{N} t) \hat{A}_{j}, j=1,2,3$ and takes the form 


$$
\begin{aligned}
& \frac{d \hat{B}_{1}}{d t}=-i \lambda_{1} \hat{B}_{2}-i \lambda_{2} \hat{B}_{3}, \\
& \frac{d \hat{B}_{2}}{d t}=-i \lambda_{1} \hat{B}_{1}, \quad \frac{d \hat{B}_{3}}{d t}=-i \lambda_{2} \hat{B}_{1} .
\end{aligned}
$$

The system (4) can be easily solved, e.g., using Laplace transformation, and the general solution is

$$
\begin{aligned}
& \hat{A}_{1}(t)=\exp (-2 i \chi \hat{N} t)\left\{\hat{a}_{1}(0) \cos \mu t-i \frac{1}{\mu}\left(\lambda_{1} \hat{a}_{2}(0)+\lambda_{2} \hat{a}_{3}(0)\right) \sin \mu t\right\}, \\
& \hat{A}_{2}(t)=\exp (-2 i \chi \hat{N} t)\left\{\left[1-2 \frac{\lambda_{1}^{2}}{\mu^{2}} \sin ^{2}\left(\frac{\mu t}{2}\right)\right] \hat{a}_{2}(0)-2 \frac{\lambda_{1} \lambda_{2} \hat{a}_{3}(0)}{\mu^{2}} \sin ^{2}\left(\frac{\mu t}{2}\right)-i \frac{\hat{a}_{1}(0) \lambda_{1}}{\mu} \sin (\mu t)\right\}, \\
& \hat{A}_{3}(t)=\exp (-2 i \chi \hat{N} t)\left\{\left[1-2 \frac{\lambda_{2}^{2}}{\mu^{2}} \sin ^{2}\left(\frac{\mu t}{2}\right)\right] \hat{a}_{3}(0)-2 \frac{\lambda_{1} \lambda_{2} \hat{a}_{2}(0)}{\mu^{2}} \sin ^{2}\left(\frac{\mu t}{2}\right)-i \frac{\lambda_{2} \hat{a}_{1}(0)}{\mu} \sin (\mu t)\right\},
\end{aligned}
$$

where $\mu=\sqrt{\lambda_{1}^{2}+\lambda_{2}^{2}}$. There are several facts which can be extracted from the structure of the Hamiltonian (1), the solution (5) and Fig. 1. When $\hat{a}_{3}(0) \leftrightarrow \hat{a}_{2}(0)$ and $\lambda_{1} \leftrightarrow \lambda_{2}$ one obtains $\hat{A}_{2}(t) \leftrightarrow \hat{A}_{3}(t)$. The nature of the coupler, i.e. the switching of energy between waveguides, manifests itself by periodic functions in (5), whereas the Kerr nonlinearities in the waveguides are described by the nonlinear (quadratic) phase, which plays an essential role in generating the nonclassical effects. On the other hand, the fundamental mode $\hat{a}_{1}$ can provide richer nonclassical effects than those produced by the second and third modes as well as the conventional coupler [21, 22] (see Fig. 1). This is resulting from the coupler mechanism, which switches the energy in the second-third mode (second waveguide) jointly to the fundamental (first waveguide) and vice versa.

On the other hand, the nature of the field quantization is evident where one can easily prove from (5) that

$$
\left[\hat{A}_{j}(t), \hat{A}_{j^{\prime}}^{\dagger}(t)\right]=\delta_{j, j^{\prime}}
$$

where $\delta_{j, j^{\prime}}$ is the Kronecker delta and $j, j^{\prime}=1,2,3$. In the derivation of (6) one has to use the identities

$$
\begin{aligned}
& \exp (-2 i \chi \hat{N} t) \hat{a}_{j}(0) \exp (2 i \chi \hat{N} t)=\hat{a}_{j}(0) \exp (2 i \chi t) \\
& \exp (-2 i \chi \hat{N} t) \hat{a}_{j}^{\dagger}(0) \exp (2 i \chi \hat{N} t)=\hat{a}_{j}^{\dagger}(0) \exp (-2 i \chi t) .
\end{aligned}
$$


Furthermore, the mean-photon number for the fundamental and second modes are:

$$
\begin{aligned}
& \hat{A}_{1}^{\dagger}(t) \hat{A}_{1}(t)=\hat{a}_{1}(0) \hat{a}_{1}^{\dagger}(0) \cos ^{2}(\mu t) \\
& +\left[\lambda_{1}^{2} \hat{a}_{2}^{\dagger}(0) \hat{a}_{2}(0)+\lambda_{2}^{2} \hat{a}_{3}^{\dagger}(0) \hat{a}_{3}(0)+\lambda_{1} \lambda_{2}\left(\hat{a}_{2}^{\dagger}(0) \hat{a}_{3}(0)+\hat{a}_{2}(0) \hat{a}_{3}^{\dagger}(0)\right)\right] \frac{\sin ^{2}(\mu t)}{\mu^{2}} \\
& +i\left[\lambda_{1}\left(\hat{a}_{1}(0) \hat{a}_{2}^{\dagger}(0)-\hat{a}_{1}^{\dagger}(0) \hat{a}_{2}(0)\right)+\lambda_{2}\left(\hat{a}_{1}(0) \hat{a}_{3}^{\dagger}(0)-\hat{a}_{1}^{\dagger}(0) \hat{a}_{3}(0)\right)\right] \frac{\sin (2 \mu t)}{2 \mu} \\
& \hat{A}_{2}^{\dagger}(t) \hat{A}_{2}(t)=\frac{\lambda_{1}^{2}}{\mu^{2}} \hat{a}_{1}^{\dagger}(0) \hat{a}_{1}(0) \sin ^{2}(\mu t)+\left[1-2 \frac{\lambda_{1}^{2}}{\mu^{2}} \hat{a}_{2}^{\dagger}(0) \hat{a}_{2}(0) \sin ^{2}\left(\frac{\mu t}{2}\right)\right]^{2}+4 \frac{\lambda_{1}^{2} \lambda_{2}^{2}}{\mu^{4}} \hat{a}_{3}^{\dagger}(0) \hat{a}_{3}(0) \sin ^{4}\left(\frac{\mu t}{2}\right) \\
& -2 \frac{\lambda_{1} \lambda_{2}}{\mu^{2}}\left[1-2 \frac{\lambda_{2}^{2}}{\mu^{2}} \sin ^{2}\left(\frac{\mu t}{2}\right)\right] \sin ^{2}\left(\frac{\mu t}{2}\right)\left[\hat{a}_{2}^{\dagger}(0) \hat{a}_{3}(0)+\hat{a}_{2}(0) \hat{a}_{3}^{\dagger}(0)\right]-\frac{i \lambda_{1}}{\mu}\left[1-2 \frac{\lambda_{2}^{2}}{\mu^{2}} \sin ^{2}\left(\frac{\mu t}{2}\right)\right] \sin (\mu t) \\
& \times\left[\hat{a}_{1}(0) \hat{a}_{2}^{\dagger}(0)-\hat{a}_{1}^{\dagger}(0) \hat{a}_{2}(0)\right]+\frac{2 i \lambda_{1}^{2} \lambda_{2}}{\mu^{3}} \sin ^{2}\left(\frac{\mu t}{2}\right) \sin (\mu t)\left[\hat{a}_{1}(0) \hat{a}_{3}^{\dagger}(0)-\hat{a}_{1}^{\dagger}(0) \hat{a}_{3}(0)\right] .
\end{aligned}
$$

From (8) the correlation between modes and switching mechanism, i.e. when one photon is created in the one of the modes the other is annihilated in the other mode, are quite obvious

We close this section by calculating the moments $\left\langle\prod_{j=1}^{3} \hat{A}_{j}^{\dagger n_{j}} \hat{A}_{j}^{m_{j}}\right\rangle$ when the modes are initially prepared in the coherent states $\left|\alpha_{1}, \alpha_{2}, \alpha_{3}\right\rangle$ and $\alpha_{j}$ are real. From (5) one can easily deduce that

$$
\left\langle\prod_{j=1}^{3} \hat{A}_{j}^{\dagger n_{j}} \hat{A}_{j}^{m_{j}}\right\rangle=\bar{\alpha}_{1}^{* n_{1}}(t) \bar{\alpha}_{1}^{m_{1}}(t) \bar{\alpha}_{2}^{* n_{2}}(t) \bar{\alpha}_{2}^{m_{2}}(t) \bar{\alpha}_{3}^{* n_{3}}(t) \bar{\alpha}_{3}^{m_{3}}(t) z^{h_{1}} \exp \left[\epsilon\left(z^{h_{2}}-1\right)\right]
$$

where $n_{j}, m_{j}$ are positive integers,

$$
\begin{aligned}
& \epsilon=\left|\alpha_{1}\right|^{2}+\left|\alpha_{2}\right|^{2}+\left|\alpha_{3}\right|^{2}, \quad z=\exp (-2 i \chi t) \\
& h_{1}=\frac{1}{2} \sum_{j=1}^{3}\left[m_{j}\left(m_{j}-1\right)-n_{j}\left(n_{j}-1\right)\right]+m_{1}\left(m_{2}+m_{3}\right)+m_{2} m_{3}-n_{1}\left(n_{2}+n_{3}\right)-n_{2} n_{3} \\
& h_{2}=m_{1}+m_{2}+m_{3}-n_{1}-n_{2}-n_{3}
\end{aligned}
$$

and

$$
\begin{aligned}
& \bar{\alpha}_{1}(t)=\alpha_{1} \cos (\mu t)-i\left[\lambda_{1} \alpha_{2}+\lambda_{2} \alpha_{3}\right] \frac{\sin (\mu t)}{\mu}=\alpha_{1 x}(t)+i \alpha_{1 y}(t), \\
& \bar{\alpha}_{2}(t)=\alpha_{2}\left[1-\frac{2 \lambda_{1}^{2}}{\mu^{2}} \sin ^{2}\left(\frac{1}{2} \mu t\right)\right]-\frac{2 \lambda_{1} \lambda_{2} \alpha_{3}}{\mu^{2}} \sin ^{2}\left(\frac{\mu t}{2}\right)-i \frac{\lambda_{1} \alpha_{1}}{\mu} \sin (\mu t)=\alpha_{2 x}(t)+i \alpha_{2 y}(t), \\
& \bar{\alpha}_{3}(t)=\alpha_{3}\left[1-\frac{2 \lambda_{2}^{2}}{\mu^{2}} \sin ^{2}\left(\frac{1}{2} \mu t\right)\right]-\frac{2 \lambda_{1} \lambda_{2} \alpha_{2}}{\mu^{2}} \sin ^{2}\left(\frac{\mu t}{2}\right)-i \frac{\lambda_{2} \alpha_{1}}{\mu} \sin (\mu t)=\alpha_{3 x}(t)+i \alpha_{3 y}(t) .
\end{aligned}
$$


Expression (9) will be frequently used in the paper. It is obvious that when $\chi t=m \pi$ and $m$ is integer, i.e. $z=1$, this expression reduces to that for the coherent light with amplitudes $\bar{\alpha}_{j}(t)$ indicating that the system produces coherent light periodically. Also from this expression one can easily check that $\left\langle\hat{A}_{j}^{\dagger m_{j}} \hat{A}_{j}^{m_{j}}\right\rangle=\left\langle\hat{A}_{j}^{\dagger} \hat{A}_{j}\right\rangle^{m_{j}}$. This means that the system is Poissonian, i.e. it cannot exhibit sub-Poissonian statistics, and the mean-photon numbers cannot provide RCP. Expression (9) reduces to that of the two-mode KNC [21] when $\lambda_{2}=0$ and $\alpha_{3}=0$. Now we use the calculations given here to investigate quadrature squeezing, Wigner function and purity in the following sections.

\section{QUADRATURE SQUEEZING}

Squeezing is a pure nonclassical phenomenon without classical analog. Squeezed light has less noise in one of the field quadrature than the vacuum level and an excess of noise in the other quadrature such that the uncertainty principle is satisfied. Squeezed light can be measured in the homodyne detection. This light has a lot of applications, e.g., in optical communication networks [29], quantum information [30] and high precision measurements. In this section we investigate the single- and two-mode squeezing and shed the light briefly on the evolution of the three-mode squeezing. In doing so we define two quadratures $\hat{X}_{n}$ and $\hat{Y}_{n}$ as

$$
\hat{X}_{n}=\frac{1}{2} \sum_{j=1}^{n}\left[\hat{A}_{j}(t)+\hat{A}_{j}^{\dagger}(t)\right], \quad \hat{Y}_{n}=\frac{1}{2 i} \sum_{j=1}^{n}\left[\hat{A}_{j}(t)-\hat{A}_{j}^{\dagger}(t)\right],
$$

where $n$ takes on values 1,2 and 3 associated with the single-, two- and three-mode squeezing, respectively. Quadratures (12) satisfy the following commutation rule:

$$
\left[\hat{X}_{n}, \hat{Y}_{n}\right]=\frac{i C_{n}}{2}
$$

where $C_{n}$ is a $c$-number and takes on the value $C_{1}=1, C_{2}=2, C_{3}=3$. The uncertainty relation associated with the commutation rule (13) is

$$
\left\langle\left(\triangle \hat{X}_{n}\right)^{2}\right\rangle\left\langle\left(\triangle \hat{Y}_{n}\right)^{2}\right\rangle \geq \frac{\left|C_{n}\right|^{2}}{16}
$$

where $\left\langle\left(\triangle \hat{X}_{n}\right)^{2}\right\rangle=\left\langle\hat{X}_{n}^{2}\right\rangle-\left\langle\hat{X}_{n}\right\rangle^{2}$ and similar form can be given for $\left\langle\left(\triangle \hat{Y}_{n}\right)^{2}\right\rangle$. The system is said to be squeezed in the $X_{n}$-quadrature if

$$
S_{n}=4\left\langle\left(\triangle \hat{X}_{n}(t)\right)^{2}\right\rangle-\left|C_{n}\right| \leq 0
$$


The equality sign in (15) holds for minimum-uncertainty states. Similar definition can be given for the $Y_{n}$-quadrature (defining a $Q_{n}$-factor). Based on (9) and (15) we investigate the single- and two-mode squeezing in details and comment on the three-mode squeezing. As we deal with the Kerr media we can expect squeezing in the guided modes in all these types.

\section{A. Single-mode squeezing}

The squeezing factors for the $j$ th mode can be straightforwardly evaluated as

$$
\begin{aligned}
& S_{1}^{(j)}(t)=2 \alpha_{j x}^{2}(t)+2 \alpha_{j y}^{2}(t)+2\left\{\left[\alpha_{j x}^{2}(t)-\alpha_{j y}^{2}(t)\right] \cos \Theta_{1}+2 \alpha_{j x}(t) \alpha_{j y}(t) \sin \Theta_{1}\right\} \exp \left[-2 \epsilon \sin ^{2}(2 \chi t)\right], \\
& -4\left[\alpha_{j x}(t) \cos \Theta_{2}+\alpha_{j y}(t) \sin \Theta_{2}\right]^{2} \exp \left[-4 \epsilon \sin ^{2}(\chi t)\right], \\
& Q_{1}^{(j)}(t)=2 \alpha_{j x}^{2}(t)+2 \alpha_{j y}^{2}(t)-2\left\{\left[\alpha_{j x}^{2}(t)-\alpha_{j y}^{2}(t)\right] \cos \Theta_{1}+2 \alpha_{j x}(t) \alpha_{j y}(t) \sin \Theta_{1}\right\} \exp \left[-2 \epsilon \sin ^{2}(2 \chi t)\right] \\
& -4\left[\alpha_{j x}(t) \sin \Theta_{2}-\alpha_{j y}(t) \cos \Theta_{2}\right]^{2} \exp \left[-4 \epsilon \sin ^{2}(\chi t)\right],
\end{aligned}
$$

where

$$
\Theta_{1}=2 \chi t+\epsilon \sin (4 \chi t), \quad \Theta_{2}=\epsilon \sin (2 \chi t) .
$$

The subscript 1 means single-mode squeezing and the superscript $j$ denotes the mode under consideration. Now we give some analytical results showing that the system can provide single-mode squeezing depending on the values of the interaction parameters. For instance, when $\left(\alpha_{1}, \alpha_{2}, \alpha_{3}\right)=(\alpha, 0,0)$ and $\chi t=m \pi / 2, m$ is odd integer, expressions (16) give for the fundamental and second modes the following:

$$
\begin{aligned}
& S_{1}^{(1)}(t)=-4 \alpha^{2} \cos ^{2}(\mu t) \exp (-4 \epsilon), \quad Q_{1}^{(1)}(t)=4 \alpha^{2} \cos ^{2}(\mu t), \\
& S_{1}^{(2)}(t)=4 \frac{\lambda_{1}^{2} \alpha^{2}}{\mu^{2}} \sin ^{2}(\mu t), \quad Q_{1}^{(2)}(t)=-4 \frac{\lambda_{1}^{2} \alpha^{2}}{\mu^{2}} \sin ^{2}(\mu t) \exp (-4 \epsilon) .
\end{aligned}
$$

It is worth reminding that the expression associated with the third mode can be obtained from that of the second mode via the transformation $\lambda_{1} \leftrightarrow \lambda_{2}$ and $\alpha_{2} \leftrightarrow \alpha_{3}$. From (18) the complementarity between the squeezing factors in the two waveguides is obvious, i.e. maximum squeezing in the first waveguide is accompanied by minimum squeezing in the 

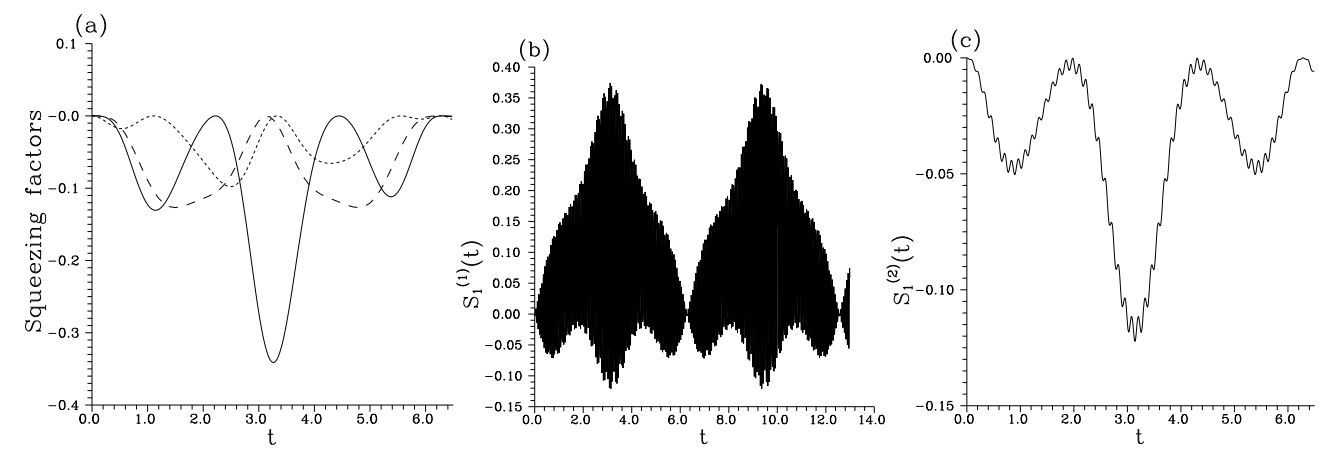

FIG. 2: Evolution of the single-mode squeezing factors for $\chi=0.5 s^{-1},\left(\alpha_{2}, \alpha_{3}\right)=(0.3,0.3)$ and for (a) $\left(\alpha_{1}, \lambda_{1}, \lambda_{2}\right)=\left(0,1 s^{-1}, 1 s^{-1}\right)$ (solid curve for $Q_{1}^{(1)}(t)$, short-dashed curve for $S_{1}^{(2)}(t)$ and longdashed curve is the squeezing factor $Q_{1}^{(1)}(t)$ for the two-mode KNC when $\left(\alpha_{1}, \alpha_{2}, \alpha_{3}, \lambda_{1}, \lambda_{2}\right)=$ $\left.\left(0,0.3 \sqrt{2} s^{-1}, 0,1 s^{-1}, 0 s^{-1}\right)\right)$, and $\left(\alpha_{1}, \lambda_{1}, \lambda_{2}\right)=\left(0.3,1 s^{-1}, 50 s^{-1}\right)$ for $(\mathrm{b}) S_{1}^{(1)}(t)$ and $(\mathrm{c}) S_{1}^{(2)}(t)$.

second waveguide and vice versa. Maximum squeezing occurs in the fundamental mode when $\mu t=m^{\prime} \pi$ and $m^{\prime}$ is integer. In this case, $S_{1}^{(1)}(t)$ can be expressed as

$$
S_{1}^{(1)}\left(t=m^{\prime} \pi / \mu\right)=-\eta \exp (-\eta), \quad \eta=4 \alpha^{2} .
$$

By evaluating the extreme values for (19) one finds that the maximum squeezing in $S_{1}^{(1)}(t)$ is $37 \%$ when $\alpha=0.5$. Also form (18) it can be proved that the maximum squeezing involved in $S_{1}^{(2)}(t)$ is the half of that of $S_{1}^{(1)}(t)$, i.e. $18.5 \%$. It is worth reminding that for the single-mode case $C_{1}=1$, i.e. $S_{1}^{(j)}(t)$ is normalized. Now we clarify the role of the switching mechanism in the behaviour of the coupler since the Kerr-like media can generate nonclassical effects regardless of entanglement between modes. This can be understood by assuming that $\lambda_{1}=\lambda_{2}=0$, i.e. the linear interaction between waveguides is neglected, and $\alpha_{1}=0$. In this case the fundamental mode cannot generate nonclassical effects, however, in the framework of coupler the fundamental mode exhibits nonclassical effects. In other words, entanglement between different components of the system can generate as well as amplify the nonclassical effects (if they exist). Information about this situation is shown in Fig. 2(a) for the given values of the parameters. It is worth mentioning that for $Q_{1}^{(1)}(t)$ of the two-mode KNC (as well as $S_{2}(t)$ in Fig. 3(a)) the initial total photon number has been considered equal to that of the three-mode KNC for significant comparison. In this case we have noted that the squeezing occurs only in the one of the quadratures, i.e. in $Y_{n}\left(X_{n}\right)$-quadrature for the fundamental (second) modes. Also squeezing generated in the 
fundamental mode is much greater than that produced in the second mode as well as in the single-mode of the two-mode KNC. Influence of the values of the linear coupling constants $\lambda_{j}$, i.e. the intensities of energy switching between the waveguides, on the evolution of the quadrature squeezing is shown in Figs. 2(b) and (c) for $S_{1}^{(1)}(t)$ and $S_{1}^{(2)}(t)$, respectively. It is worth mentioning that $Q_{1}^{(1)}(t)$ provides a behaviour typical to that shown in Fig. 2(b). From Fig. 2(b) one can see that additionally to the occurrence of the nonclassical squeezing the $S_{1}^{(1)}(t)$ exhibits leaf-revival-collapse phenomenon. Actually, there are two requirements for occurring such phenomenon, which are: (i) The linear and nonlinear couplings in the Hamiltonian (1) should contribute significantly to the dynamics of the system. (ii) The energy exchange between the mode under consideration with at least one of the modes in the other waveguide is very strong. With this in mind we can understand why $S_{1}^{(2)}(t)$ provides squeezing rather than RCP. This can be realized easily when $\lambda_{2}>>\lambda_{1}$, i.e. $\lambda_{1} / \lambda_{2} \simeq 0$, $\bar{\alpha}_{2}(t)=\alpha$ and hence the time evolution of $S_{1}^{(2)}(t)$ basically depends on the contribution of the Kerr-like medium, which alone cannot produce RCP. Generally, the behaviour of the system for this case can be explained as follows. As $\lambda_{2}>>\lambda_{1}$, i.e. the energy switching mainly occurs between the fundamental and third modes, which behave as a two-mode KNC and the second mode practically does not affect the behaviour. Consequently, the second mode behaves as an independent mode evolving in a Kerr medium. In conclusion, when $\lambda_{1}$ and $\lambda_{2}$ are large or $\lambda_{1}>\lambda_{2}$ the squeezing factors for the fundamental and second modes provide RCP. On the other hand, the occurrence of the RCP in the squeezing factors of the fundamental mode can be explained in the following sense [21]. The squeezing factors (16) include two forms of periodic functions: one is coming from the self-cross-nonlinear interaction part of (10), in particular, the exponential function whose period is $\pi / \chi$, and the other is arising from the linear-interaction part whose period is $\pi / \mu$. When the linear interaction between waveguides is very strong-either between the fundamental-second or fundamental-third or both-the period of the energy exchange between waveguides decreases, i.e. many oscillations occur, till the interaction time becomes $t=\pi / \chi$, at this moment the field is trapped instantaneously by nonlinearity in the waveguides and the squeezing factors show collapse. The oscillations of the RCP are caused by the linear coupling $\lambda_{j}$ and the envelope of the revivals is caused by the nonlinearity in the system. As the interaction proceeds the phenomenon is periodically repeated.

We have to stress that the evolution of the squeezing factors is sensitive to the values of 
the intensities, however, in the strong-intensity regime it can provide nonclassical squeezing instantaneously greater than that present in Figs. 2, but the behaviour becomes rather complicated. We draw the attention to this point in the following section.

\section{B. Two-mode squeezing}

In this part we study the two-mode squeezing in which the correlation between modes starts to play a role. Also we compare the behaviour here with the corresponding one for the two-mode KNC. We restrict the analysis to the fundamental-second (between waveguides) and second-third modes (in the same waveguide). The fundamental-second mode squeezing factors can be straightforwardly evaluated as

$$
\begin{aligned}
& S_{2}^{(1,2)}(t)=\frac{1}{2}\left[S_{1}^{(1)}(t)+S_{1}^{(2)}(t)\right]+2\left\{\left[\alpha_{1 x}(t) \alpha_{2 x}(t)-\alpha_{1 y}(t) \alpha_{2 y}(t)\right] \cos \Theta_{2}\right. \\
& \left.+\left[\alpha_{2 x}(t) \alpha_{1 y}(t)+\alpha_{1 x}(t) \alpha_{2 y}(t)\right] \sin \Theta_{2}\right\} \exp \left[-2 \epsilon \sin ^{2}(2 \chi t)\right]+2\left[\alpha_{1 x}(t) \alpha_{2 x}(t)+\alpha_{1 y}(t) \alpha_{2 y}(t)\right] \\
& -4\left[\alpha_{1 x}(t) \cos \Theta_{1}+\alpha_{1 y}(t) \sin \Theta_{1}\right]\left[\alpha_{2 x}(t) \cos \Theta_{1}+\alpha_{2 y}(t) \sin \Theta_{1}\right] \exp \left[-4 \epsilon \sin ^{2}(\chi t)\right] \\
& Q_{2}^{(1,2)}(t)=\frac{1}{2}\left[Q_{1}^{(1)}(t)+Q_{1}^{(2)}(t)\right]-2\left\{\left[\alpha_{1 x}(t) \alpha_{2 x}(t)-\alpha_{1 y}(t) \alpha_{2 y}(t)\right] \cos \Theta_{2}\right. \\
& \left.+\left[\alpha_{2 x}(t) \alpha_{1 y}(t)+\alpha_{1 x}(t) \alpha_{2 y}(t)\right] \sin \Theta_{2}\right\} \exp \left[-2 \epsilon \sin ^{2}(2 \chi t)\right]+2\left[\alpha_{1 x}(t) \alpha_{2 x}(t)+\alpha_{1 y}(t) \alpha_{2 y}(t)\right] \\
& -4\left[\alpha_{1 y}(t) \cos \Theta_{1}-\alpha_{1 x}(t) \sin \Theta_{1}\right]\left[\alpha_{2 y}(t) \cos \Theta_{1}-\alpha_{2 x}(t) \sin \Theta_{1}\right] \exp \left[-4 \epsilon \sin ^{2}(\chi t)\right]
\end{aligned}
$$

where $S_{1}^{(j)}$ and $Q_{1}^{(j)}$ are given by (16) and $\Theta_{j}$ have the same meaning as given above. The subscript 2 stands for two-mode squeezing and the superscript $(1,2)$ denotes fundamentalsecond squeezing factor. The second-third squeezing factors can be easily obtain from (20) by simply replacing 1 by 3 . As we did above we analyse the case $\left(\alpha_{1}, \alpha_{2}, \alpha_{3}\right)=(\alpha, 0,0)$ and $\chi t=m \pi / 2, m$ is odd integer, for which expressions (20) reduce to

$$
\begin{aligned}
& S_{2}^{(1,2)}(t)=2 \alpha^{2}\left[\frac{\lambda_{1}^{2}}{\mu^{2}} \sin ^{2}(\mu t)-\cos (\mu t) \exp (-4 \epsilon)\right], \\
& Q_{2}^{(1,2)}(t)=2 \alpha^{2}\left[\cos (\mu t)-\frac{\lambda_{1}^{2}}{\mu^{2}} \sin ^{2}(\mu t) \exp (-4 \epsilon)\right] .
\end{aligned}
$$

Squeezing can be periodically realized in the two quadratures for particular values of interaction time. The two-mode squeezing between the second and third modes, i.e. modes 

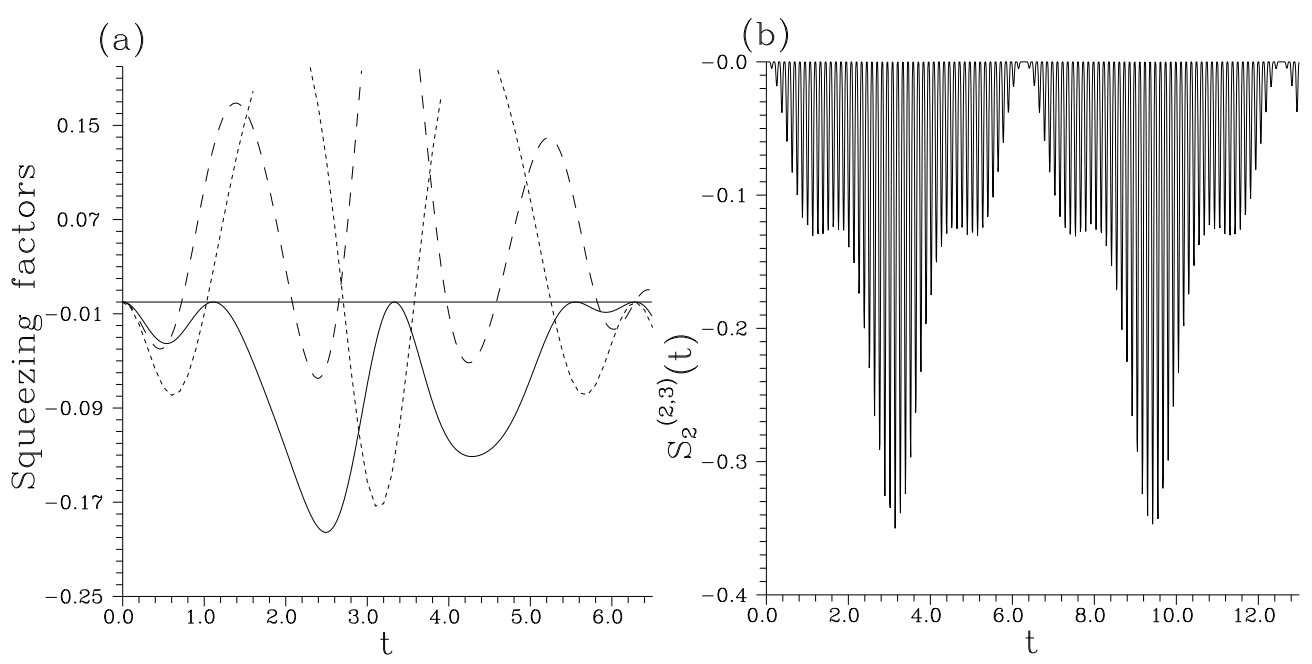

FIG. 3: Evolution of the two-mode squeezing factors for $\chi=0.5 s^{-1},\left(\alpha_{1}, \alpha_{2}, \alpha_{3}\right)=(0,0.3,0.3)$ and for (a) $\lambda_{1}=\lambda_{1}=1 s^{-1}$ (long-dashed curve for $S_{1}^{(1,2)}(t)$, solid curve for $S_{2}^{(2,3)}(t)$, the short-dashed curve is given for $S_{2}(t)$ factor of the two-mode $\mathrm{KNC}$ when $\left(\alpha_{1}, \alpha_{2}, \alpha_{3}, \lambda_{1}, \lambda_{2}\right)=$ $\left.\left(0,0.3 \sqrt{2}, 0,1 s^{-1}, 0 s^{-1}\right)\right)$ and the straight line is given for showing the squeezing bound), (b) $S_{2}^{(2,3)}(t)$ for $\lambda_{1}=50 s^{-1}, \lambda_{2}=1 s^{-1}$.

evolve in the same waveguide, for this special case is

$$
\begin{aligned}
& S_{2}^{(2,3)}(t)=2 \alpha^{2} \frac{\left(\lambda_{1}+\lambda_{2}\right)^{2}}{\mu^{2}} \sin ^{2}(\mu t), \\
& Q_{2}^{(2,3)}(t)=-2 \alpha^{2} \frac{\left(\lambda_{1}+\lambda_{2}\right)^{2}}{\mu^{2}} \sin ^{2}(\mu t) \exp (-4 \epsilon) .
\end{aligned}
$$

Squeezing can occur in the second quadrature only similar to the single-mode case. Now we draw the attention to the behaviour of the general forms (20), which are presented in Figs. 3(a) and (b) for given values of the interaction parameters. From Fig. 3(a) one can observe that $S_{2}^{(1,2)}(t)$ and $S_{2}(t)$ exhibit nonclassical squeezing periodically. Complementary behaviours have been noticed for $Q_{2}^{(1,2)}(t)$ and $Q_{2}(t)$. Nevertheless, for modes evolving in the same waveguide where switching mechanism does not exist directly, squeezing occurs-for these values of interaction parameters-only in one of the two quadratures, i.e. $S_{2}^{(2,3)}(t)$, as indicated by the solid curve. We have to stress that the three-mode KNC provides amount of squeezing in the framework of two-mode squeezing greater than that of the two-mode KNC (compare the solid curve with the short-dashed curve). The action of the coupling constant is shown in Fig. 3(b) for $S_{2}^{(2,3)}(t)$. From this figure it is obvious that squeezing 
(or minimum-uncertainty light) always occurs and the amount of squeezing is greater than that obtained for weak-coupling case (compare with the solid curve in Fig. 3(a)). This means that the linear mechanism in the system can play a significant role in amplifying the nonclassical effects. RCP is remarkable, which always occurs provided that $\lambda_{1}$ or $\lambda_{2}$ or both are large. Also for these cases we have noted that the two-mode squeezing factors between the waveguides exhibit RCP somewhat similar to that shown in Fig. 2(b).

Finally, for the three-mode squeezing factors we have noted that squeezing periodically occurs in the two quadratures similar to that of the two-mode case, in particular, the fundamental-second mode factors. Moreover, the amount of squeezing achieved in the threemode factors are greater than those in the single-mode and two-mode factors. Also intense switching between the waveguides provide RCP in the evolution of the three-mode squeezing factors.

\section{QUASIPROBABILITY DISTRIBUTION FUNCTION}

Quasiprobability distribution functions, namely, Wigner $W$-, Husimi $Q$ - and Glauber $P$-functions [31], provide all physical information about the quantum mechanical systems. Investigation of these functions for the quantum mechanical systems is one of the main topics in quantum optics. Actually, among all quasiprobability functions $W$ function takes a considerable interest since it has been experimentally realized by different techniques, e.g. in homodyne tomography [32], photon counting experiment [33] and in experiments with trapped ions [34], and it is sensitive to the interference in phase space. In this section we investigate the single-mode quasiprobability distribution functions, in particular, $W$ function for the system under consideration. As the derivations of these functions are similar with those in [21], we write down only the explicit formulae for them. Just here we give only outline of the derivation. The solution (5) includes exponential quadratic operator phase, which causes difficulties in evaluating the quasiprobability functions in the standard way. Thus one has to use the technique given in [35], which basically depends on the formula (9), to achieve the goal. It is worth reminding that the modes are initially prepared in the coherent state $\left|\alpha_{1}, \alpha_{2}, \alpha_{3}\right\rangle$. Furthermore, we extend the investigation to include the purity for the single-mode case, which can be evaluated by means of the symmetric characteristic function. Now the $j$ th mode symmetric characteristic function is [21] 

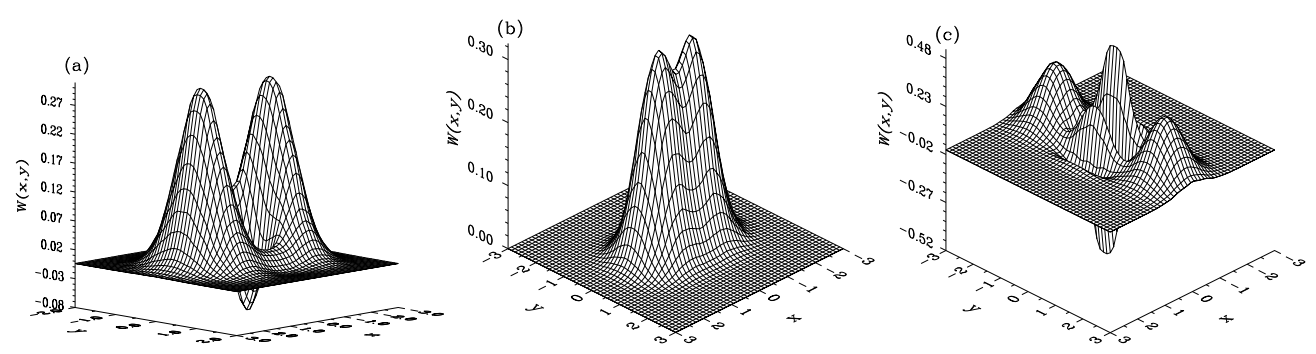

FIG. 4: The $W$ function for the single-mode case for $\chi=0.5 s^{-1}, t=\pi s$ and for $\alpha_{j}=0.9, \lambda_{1}=$ $\lambda_{2}=1 /\left(2 \sqrt{2} s^{-1}\right.$ ) (a) (fundamental mode), (b) (second mode); and $\alpha_{1}=2, \alpha_{2}=\alpha_{3}=0$ and $\lambda_{1}=\lambda_{2}=1 / \sqrt{2} s^{-1}$ (c) (second mode).

$$
\begin{aligned}
& C_{j}(\zeta, t)=\exp \left(\frac{-1}{2}|\zeta|^{2}\right) \sum_{n_{1}, n_{2}=0}^{\infty} \frac{\zeta^{n_{1}}\left(-\zeta^{*}\right)^{n_{2}}}{n_{1} ! n_{2} !} \bar{\alpha}_{j}^{n_{2}}(t) \bar{\alpha}_{j}^{* n_{1}}(t) \\
& \times z^{\left[\frac{n_{2}}{2}\left(n_{2}-1\right)-\frac{n_{1}}{2}\left(n_{1}-1\right)\right]} \exp \left[\epsilon\left(z^{n_{2}-n_{1}}-1\right)\right] .
\end{aligned}
$$

Also the $W$ function for the $j$ th mode takes the form [21]:

$$
\begin{aligned}
& W_{j}(\beta, t)=\frac{2}{\pi} \exp \left(-2|\beta|^{2}\right) \sum_{n_{1}, n_{2}=0}^{\infty} \frac{(-1)^{n_{1}} \bar{\alpha}_{j}^{n_{2}}(t) \bar{\alpha}_{j}^{* n_{1}}(t)}{n_{2} !} 2^{n_{2}} \beta^{* n_{2}-n_{1}} \\
& \times z^{\left[\frac{n_{2}}{2}\left(n_{2}-1\right)-\frac{n_{1}}{2}\left(n_{1}-1\right)\right]} \exp \left[\epsilon\left(z^{n_{2}-n_{1}}-1\right)\right] \mathrm{L}_{n_{1}}^{n_{2}-n_{1}}\left(2|\beta|^{2}\right),
\end{aligned}
$$

where $\mathrm{L}_{n}^{m}($.$) is the associated Laguerre polynomial of order n$ and $\beta=x+i y$. One can easily verify that when $\chi t=m^{\prime} \pi$ and $m^{\prime}$ is integer, the system produces coherent light with amplitude $\bar{\alpha}_{j}(t)$ [21]. Furthermore, for $t \chi=\left(m^{\prime}+1 / 2\right) \pi$ and $m^{\prime}$ is integer, expression (24) gives that for the cat states (the derivation is given in the appendix of [21])

$$
\begin{aligned}
& W_{j}(\beta, t)=\frac{1}{\pi}\left\{\exp \left(-2\left|\beta-i \bar{\alpha}_{j}(t)\right|^{2}\right)+\exp \left(-2\left|\beta+i \bar{\alpha}_{j}(t)\right|^{2}\right)\right. \\
& \left.+2 \exp \left[-2\left(|\beta|^{2}+D_{j}\right)\right] \sin \left(2\left(\beta \bar{\alpha}_{j}^{*}(t)+\beta^{*} \bar{\alpha}_{j}(t)\right)\right)\right\},
\end{aligned}
$$

where

$$
D_{j}=\epsilon-\left|\bar{\alpha}_{j}(t)\right|^{2}
$$

Roughly speaking, this form tends to that of the YSCS [26] in the $j$ th mode when $D_{j} \simeq 0$ and $\bar{\alpha}_{j}(t) \neq 0$. In contrast to the two-mode KNC, the fundamental and second (or third) 
modes of the three-mode KNC can provide different types of cat states for certain choice of the interaction parameters. Now we give some analytical facts related to $D_{j}$. There are three different special cases, which can be discussed for (25) based on the values of $\alpha_{j}$ where we assume $\lambda_{1}=\lambda_{2}$.

(i) When $\alpha_{j}=\alpha, j=1,2,3$ for the fundamental and second modes, we obtain

$$
\begin{aligned}
& D_{1}=\alpha^{2}\left[1+\cos ^{2}(\mu t)\right], \\
& D_{2}=\alpha^{2}\left[2+\frac{1}{2} \sin ^{2}(\mu t)\right] .
\end{aligned}
$$

Expressions (27) show that $D_{2} \geq D_{1}$ and $D_{j} \simeq 0$ only when $\alpha<1$. This means that the cat states can be generated in the first waveguide faster than that in the second waveguide. As $\alpha$ is relatively small the microscopic cat states [35] can be generated in the two waveguides simultaneously. Trivially, statistical-mixture coherent states can be generated for this case in dependence on the values of the interaction parameters.

(ii) For $\left(\alpha_{1}, \alpha_{2}, \alpha_{3}\right)=(\alpha, 0,0)$ we obtain

$$
\begin{aligned}
& D_{1}=\alpha^{2} \sin ^{2}(\mu t), \\
& D_{2}=\alpha^{2}\left[1-\frac{1}{2} \sin ^{2}(\mu t)\right] .
\end{aligned}
$$

Expressions (28) indicate that when the fundamental mode provides YSCS in a macroscopical form $\alpha>1$, say, when $\mu t=\pi$, the second (or the third) produces statistical-mixture coherent states, however, when $\alpha<1$, YSCS in the microscopic forms are simultaneously generated in the two waveguides. This shows the crucial role for the intensities of the initial light.

(iii) For $\left(\alpha_{1}, \alpha_{2}, \alpha_{3}\right)=(0, \alpha, 0)$ we obtain

$$
\begin{aligned}
& D_{1}=\alpha^{2}\left[1-\frac{1}{2} \sin ^{2}(\mu t)\right] \\
& D_{2}=2 \alpha^{2}\left[1-\frac{1}{2} \sin ^{2}\left(\frac{\mu}{2} t\right)\right] \sin ^{2}\left(\frac{\mu}{2} t\right) .
\end{aligned}
$$

This case represents the inverse of the case (ii). The above cases lead to an important fact: In order to obtain macroscopically distinguishable YSCS from a particular mode it should 
be initially prepared in coherent state with large intensity. Information on the above cases is shown in Figs. 4 for the fundamental and second modes for given values of interaction parameters. The values of the linear constants $\lambda_{j}$ have been chosen to minimize $D_{1}$. In Fig. 4(a) for the fundamental mode one can observe the two Gaussian peaks and inverted negative peak inbetween indicating the occurrence of the interference in phase space. Fig. 4(b) (for the second mode) provides information on the statistical-mixture coherent states, i.e. it shows two-peak forms without interference in phase space. We have to stress that in Figs. 4(a) and (b) the states generated are close to those of the microscopic regime. Fig. $4(\mathrm{c})$ gives the well-known shape for the $W$ function of the macroscopically distinguishable YSCS. In this case the fundamental mode collapses to the state:

$$
\left|\psi_{1}(t)\right\rangle=\frac{1}{\sqrt{2}}\left[\left|i \bar{\alpha}_{1}(t)\right\rangle+\exp \left(i \frac{\pi}{2}\right)\left|-i \bar{\alpha}_{1}(t)\right\rangle\right]
$$

The $W$ function for the second mode corresponding to Fig. 4(c) is that of the vacuum state, where $\bar{\alpha}_{2}(t) \simeq 0$. Actually, we have noted that such behaviours of the fundamental and second modes-related to Fig. 4(c) and $W$ function of vacuum light-can be interchanged when one uses $t=\pi s, \alpha_{1}=\alpha_{3}=0, \alpha_{2}=2, \lambda_{1}=\lambda_{2}=\sqrt{2} s^{-1}$.

In Figs. 5 we illustrate the general case, when the above special cases are no longer applicable. In these figures we consider $t=12.38 \mathrm{~s}$, where squeezing is well pronounced in the behaviours of the single-mode case for the fundamental and second modes. In these figures the nonclassical effects are presented as asymmetric multi-peak structure indicating generation of the multi-component cat states. Also Fig. 5(a) includes additionally negative values showing that the fundamental mode gives amount of the nonclassical behaviour greater than that for the second (or third) mode. This agrees with the explanation given in section 2. On the other hand, when $\lambda_{1}>>\lambda_{2}$ these negative values disappear and the $W$ functions of the fundamental and second modes are almost the same (see Fig. 5(c)). It is worthwhile mentioning that the corresponding $W$ function for the two-mode KNC gives two symmetric peaks without involving any negative values, which is insensitive to the values of $\lambda_{1}(\geq 1)$. In conclusion, the system can generate different types of YSCS depending on the values of the interaction parameters.

Entanglement characterizes intrinsically quantum-mechanical correlations between quantum systems. In this regard quantum entanglement is the basic resource required to implement several of the most important processes studied by quantum information theory. 

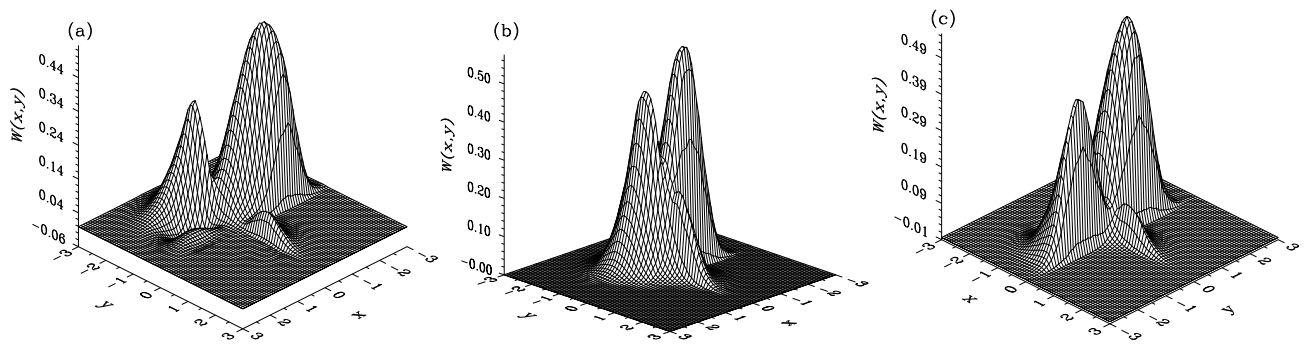

FIG. 5: The $W$ function for $\chi=0.5 s^{-1}, t=12.38 s, \alpha_{j}=1, \lambda_{1}=\lambda_{2}=1 s^{-1}$ and for (a) the fundamental mode, (b) second mode. Figure (c) is given for the fundamental mode for the case $\lambda_{1}=50 \mathrm{~s}^{-1}, \lambda_{2}=1 \mathrm{~s}^{-1}$, the values of the others parameters are the same as for (a).

Thus, we discuss the single-mode degree of purity $\operatorname{Tr} \hat{\rho}_{j}^{2}(t)$ for the system under consideration, which gives globally information about the entanglement. Purity can be determined by the knowledge of quantum state of the system, which in turn can be obtained by quantum tomography [32]. Also it is worth mentioning that in [36] entanglement has been studied for the two-mode KNC driven by external classical field showing that the system is able to generate Bell-like states. Also in [37] the purity and the relative entropy are investigated for the pumped dissipative nonlinear oscillator including Kerr nonlinearity. The purity can be evaluated by means of the symmetric characteristic function through the relation

$$
\operatorname{Tr} \hat{\rho}_{j}^{2}(t)=\frac{1}{\pi} \int\left|C_{j}(\zeta, t)\right|^{2} d^{2} \zeta
$$

When $\operatorname{Tr} \hat{\rho}_{j}^{2}(t)=1$ the mode under consideration is in a pure state, i.e. the mode under consideration is disentangled from the rest of the system, while when $\operatorname{Tr} \hat{\rho}_{j}^{2}(t)<1$ the mode is in a mixed state and consequently it is entangled with the rest of the system. The subsystems are most entangled when their reduced density matrices are maximally mixed. Substituting (23) into (31) and after lengthy but straightforward calculation we arrive at

$$
\begin{aligned}
& \operatorname{Tr} \hat{\rho}_{j}^{2}(t)=\sum_{n_{1}, m_{1}=0}^{\infty} \frac{\left(n_{1}+m_{1}\right) !(-1)^{m_{1}+n_{1}}\left|\bar{\alpha}_{j}(t)\right|^{2\left(n_{1}+m_{1}\right)}}{\left[n_{1} ! m_{1} !\right]^{2}} \\
& +2 \sum_{m_{1}>m_{2}}^{\infty} \sum_{n_{1}=0}^{\infty} \frac{\left(n_{1}+m_{1}\right) !(-1)^{n_{1}+m_{2}}\left|\bar{\alpha}_{j}(t)\right|^{2\left(n_{1}+m_{1}\right)}}{n_{1} ! m_{1} ! m_{2} !\left(n_{1}+m_{1}-m_{2}\right) !} \exp \left[-4 \epsilon \sin ^{2}\left(\left(m_{2}-m_{1}\right) \chi t\right)\right] \cos \psi\left(n_{1}, m_{1}, m_{2}\right),
\end{aligned}
$$

where

$$
\psi\left(n_{1}, m_{1}, m_{2}\right)=2 \chi t\left(m_{2}-m_{1}\right)\left(m_{2}-n_{1}\right) .
$$



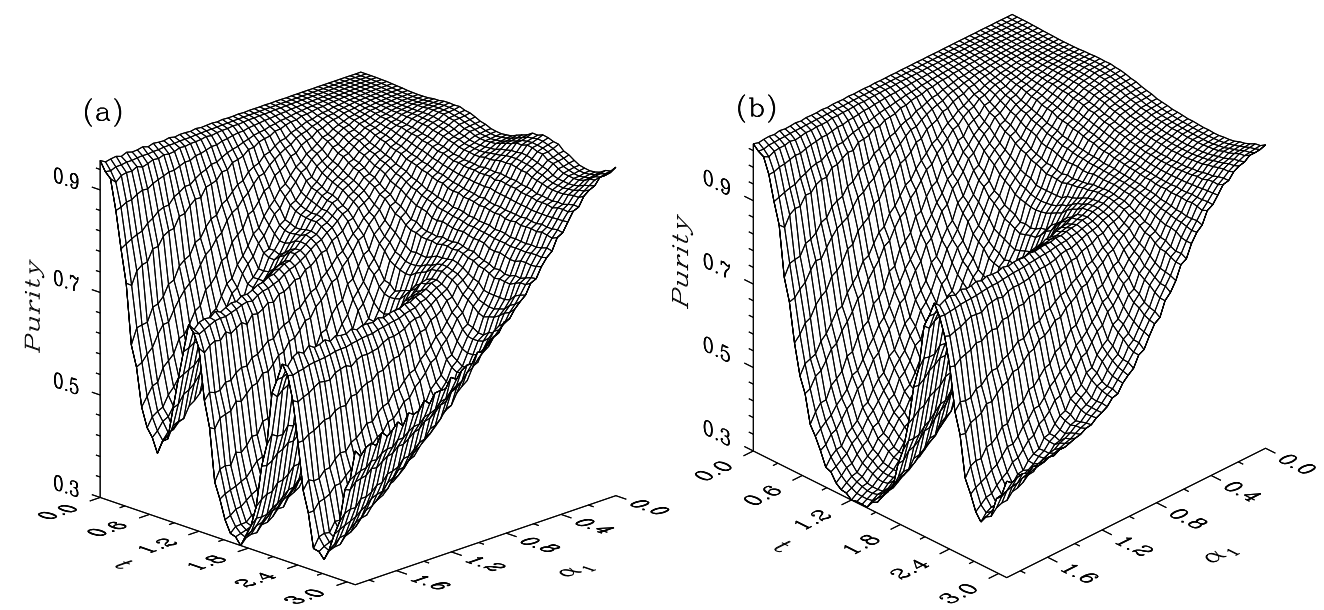

FIG. 6: The evolution of the purity $\operatorname{Tr} \hat{\rho}_{j}^{2}(t)$ for $\chi=0.5 s^{-1}, \alpha_{1}=\alpha_{2}=0.3$ and $\lambda_{1}=\lambda_{2}=1 s^{-1}$ for the fundamental mode (a) and the second mode (b).

It is evident that (32) is dependent on the mean-photon number for the mode under consideration. Thus for fixed value of $\chi$ and $\chi t \neq m \pi, \quad m$ is integer, the evolution of the purity reflects well the switching mechanism of the coupler provided that at least one of the initial intensities is large. In relation to Figs. 6, i.e. $\lambda_{1}=\lambda_{2}, \alpha_{2}=\alpha_{3}=\alpha$, the mean-photon numbers for the fundamental and second modes take the forms

$$
\left|\bar{\alpha}_{1}(t)\right|^{2}=\alpha_{1}^{2} \cos ^{2}(\mu t)+2 \alpha^{2} \sin ^{2}(\mu t), \quad\left|\bar{\alpha}_{2}(t)\right|^{2}=\alpha^{2} \cos ^{2}(\mu t)+\frac{\alpha_{1}^{2}}{2} \sin ^{2}(\mu t) .
$$

When $\alpha_{j}$ are small, the mean-photon numbers of the fundamental and second modes would be relatively small and hence the evolution of the purity will be close to that of the initial case (see Figs. 6(a) and (b) for given values of the parameters). Also from Figs. 6 we note that the fundamental and second modes tend to partial pure states, i.e. close to pure states, when their mean-photon numbers provide their initial values. In this case the components of the system are approximately disentangled. Additionally, the fundamental mode only tends to almost pure state when the energies in the two waveguides are completely interchanged. More illustratively, from these figures one can observe that when $t=0 \mathrm{~s}$ the two modes are in pure states. When $t$ increases and $\alpha_{1}$ is large, entanglement between modes through the interaction (1) is achieved and both the fundamental and second modes become mixed. The fundamental mode provides first maximum mixedness at $t \simeq 0.69 \mathrm{~s}$ (see Fig. 6(a)). Actually, at this value of the interaction time, i.e. $t \simeq 0.69 \mathrm{~s}$, the mean-photon numbers for the three modes are equal and consequently their purities are equal, i.e. $\operatorname{Tr} \hat{\rho}_{j}^{2}(t) \simeq 0.37$ (we have 
checked these facts). When the interaction proceeds, switching energy between waveguides continues until $t \mu=\pi / 2$ then the energies in the waveguides are completely interchanged (cf. (34) ). At this moment the fundamental (second) mode is in partial pure (maximum mixed) state since its mean-photon number is small (large). This behaviour is repeated until $\mu t=\pi$ where the fundamental and second modes provide their initial mean-photon numbers.

Now we draw the attention to find analytically the values of the interaction time for which the modes of the system are completely disentangled, i.e. $\operatorname{Tr} \hat{\rho}_{j}^{2}(t)=1$. This can be realized from (91) since all calculations in the paper depend on this expression. Therefore the condition for disentanglement is that

$$
\left\langle\prod_{j=1}^{3} \hat{A}_{j}^{\dagger n_{j}} \hat{A}_{j}^{m_{j}}\right\rangle=\prod_{j=1}^{3}\left\langle\hat{A}_{j}^{\dagger n_{j}} \hat{A}_{j}^{m_{j}}\right\rangle .
$$

From the analysis given in the paper this occurs when $\chi t=m \pi, m$ is integer. In this case the modes are completely disentangled and each of which has a coherent light with time-dependent amplitude. On the other hand, the values of interaction times for which the system provides its initial light can be obtained by solving simultaneously the following two equations

$$
\mu t=2 m^{\prime} \pi, \quad \chi t=m \pi,
$$

where $m^{\prime}$ and $m$ are integers. These equations are connected with the requirements that $\bar{\alpha}_{j}(t)=\alpha_{j}$. Equations (36) lead to the fact that the system tends to the initial stage when $\mu=\chi$ and $\mu t=2 m^{\prime} \pi$. In this case the linearity of the system compensates the nonlinearity.

\section{CONCLUSION}

In this paper we have discussed the quantum properties for the three-mode codirectional nonlinear Kerr coupler, when the modes are initially prepared in coherent light. After obtaining the exact solution of equations of motion we have investigated single- and two-mode quadrature squeezing, $W$ function and purity. Various interesting effects have been obtained. Different forms of squeezing have been achieved by varying the parameters $\chi, \lambda_{j}$ and $\alpha_{j}$. Squeezing can be equally shared between the guided modes and the interacting modes can behave as two separated dynamical systems by controlling the intensity of switching between waveguides. Quadrature squeezing can exhibit leaf-revival-collapse phenomenon based 
on the competition between the linearity and nonlinearity in the system. Moreover, different forms of cat states have been generated and confirmed in the evolution of $W$ function. We have analytically proved that two different types of cat states can be simultaneously realized in the evolution of the fundamental and second (third) modes. Also we have discussed the mixedness for the single-mode case and deduced the values of the interaction time as well as the conditions required for complete disentanglement between the components of the system. The nature of the coupler has manifested itself in all studied quantities as complementary between the behaviours of the fundamental and second-third modes, i.e. as switching energy between waveguides. Moreover, we have showed that the nonclassical effects provided by the fundamental mode are richer than those for both the second and third modes as well as for the two-mode KNC. Finally, the system discussed in this paper is more effective than the conventional Kerr coupler and can be used to amplify the nonclassical effects.

\section{Acknowledgement}

J.P. and F.A.A.E. thank the partial support from the grant LN00A015 of the Czech Ministry of Education and from the EU Project COST OCP 11.003.

\section{References}

[1] Jensen S M 1982 IEEE J. Quant. Electron. QE 181580.

[2] Ekert A and Jozsa R 1996 Rev. Mod. Phys. 68 733; Lo H-K, Popescu S and Spiller T 1998 " Introduction to Quantum Computation and Information" (World Scientific: Singapore); Begie A, Braun D, Tregenna B and Knight P L 2000 Phys. Rev. Lett. 851762.

[3] Assanto G, Stegeman G I, Sheik-Bahae M and Van Stryland E W 1993 Appl. Phys. Lett. 62 1323; Karpierz M, Wolinski T and Swillo M 1996 Molec. Crystals Liq. Crystals 282365.

[4] Chefles A and Barnett S M 1996 J. Mod. Opt. 43709.

[5] Townsend P D, Baker G L, Shelburne J L III and Etemad S 1989 Proc. SPIE 1147256.

[6] Fazio E, Sibilia C, Senesi F and Bertolotti M 1996, Opt. Commun. 127 62; Brooks D, Ruschin S and Scarlat D 1996, IEEE J. Selected Topics in Quant. Electr. 2 210; Luff B J, Harris R D, 
Wilkinson J S, Wilson R and Schiffrin D J 1996, Opt. Lett. 21 618; Guo A, Henry M, Salamo G J, Segev M and Wood G L 2001, Opt. Lett. 26 1274; Wang Q and He S 2002 IEEE J. Select. Topics in Quant. Electr. 8 1233; Zhou B, Xu C-Q and Chen B 2003, J. Opt. Soc. Am. $B 20846$.

[7] Finlayson N, Banyai W C, Wright E M, Seaton C T, Stegeman G I, Cullen T J and Ironside C N 1988, Appl. Phys. Lett. 531144.

[8] Soldano L B and Pennings E C M 1995, J. Lightwave Technol. 13615.

[9] Tricca D, Sibilia C, Severini S, Bertolotti M, Scalora M, Bowden C M and Sakora K 2004, J. Opt. Soc. Am. B 21671.

[10] Banaszek K, U'Ren A B and Wamsley I A 2001, Opt. Lett. 261367.

[11] Peřina J and Peřina J Jr 1996 J. Mod. Opt. 43 1951; Abdalla M S, El-Orany F A A and Peřina J 1999 J. Phys. A: Math. Gen. 32 3457; Abdalla M S, El-Orany F A A and Peřina J 2000 J. Mod. Opt. 471055.

[12] Peřina J and Peřina J Jr 1995 Quant. Semiclass. Opt. 7 541; Peřina J 1996 J. Mod. Opt. 42 1517; El-Orany F A A, Peřina J and Abdalla M S 2001 Quant. Semiclass. opt. 3 67; Ibid 2001 Phys. Scr. 63 128; Ibid 2001 Inter. J. Mod. Phys. B 152125.

[13] Fiurášek J and Peřina J 1999 J. Mod. Opt. 461255.

[14] Mogilevtsev D, Korolkova N and Peřina J 1997 J. Mod. Opt. 441293.

[15] Korolkova N and Peřina J 1997 Opt. Commun. 136135.

[16] Korolkova N and Peřina J 1997 J. Mod. Opt. 441525.

[17] Fiurášek J, Křepelka J and Peřina J 1999 Opt. Commun. 167115.

[18] Ibrahim A-B M A, Umarov B A and Wahiddin M R B 2000 Phys. Rev. A 61043804.

[19] Ariunbold G and Peřina J 2000 Opt. Commun. 176149.

[20] Ariunbold G and Peřina J 20002001 J. Mod. Opt. 481005.

[21] El-Orany F A A, Abdalla M S and Peřina J 2004 J. Opt. B: Quan. Semiclass. Opt. 6460.

[22] El-Orany F A A and Peřina J 2004 Phys. Lett. A 333204.

[23] Peřina Jr J and Peřina J 2000 Progress in Optics 41, ed. E. Wolf (Amsterdam: Elsevier), p. 361; Fiurášek J and Peřina J 2001 Coherence and Statistics of Photons and Atoms, ed. J. Peřina (New York: J. Wiley), p. 65.

[24] Silberhorn C, Lam P K, WeiB O, König F, Korolkova N and Leuchs G 2001 Phys. Rev. Lett. 864267. 
[25] Fanjoux G, Devaux F, Lantz E and Maillotte H 2004, J. Opt. B: Quant. Semiclass. Opt. 6 S250.

[26] Yurke B and Stoler D 1986 Phys. Rev. Lett. 5713.

[27] Bužek V 1989 Phys. Rev. A 39 5432; Miranowicz A, Tanaś R and Kielich S 1990 Quant. Opt. 2 253; Miranowicz A, Tanaś R, Gantsog Ts and Kielich S 1991 J. Opt. Soc. Am. B 81576.

[28] W. Louisell, Radiation and Noise in Quantum Electronics (McGraw-Hill, New York, 1964), p. 274 .

[29] Yuen H P and Shapiro J H 1980 IEEE Trans. Inform. Theory IT26 78.

[30] Braunstein S L and Kimble H J 1998 Phys. Rev. Lett. 80 869; Milburn G J and Braunstein S L 1999 Phys. Rev. A. 60 937; Ralph T C 2000 Phys. Rev. A 61 010303(R); Hillery M 2000 Phys. Rev. A 61022309.

[31] Wigner E 1932 Phys. Rev. 40 749; Cahill K E and Glauber R J 1969 Phys. Rev. 177 1882; Hillery M, O’Connell R F, Scully M O and Winger E P 1984 Phys. Rep. 106121.

[32] Beck M, Smithey D T and Raymer M G 1993 Phys. Rev. A 48 890; Smithey D T, Beck M, Cooper J and Raymer M G 1993 Phys. Rev. A 48 3159; Beck M, Smithey D T, Cooper J and Raymer M G 1993 Opt. Lett. 18 1259; Smithey D T, Beck M, Cooper J, Raymer M G and Faridani M B A 1993 Phys. Scr. T 48 35. Leonhardt U 1997 "Measuring the Quantum State of Light" (University Press: Cambridge).

[33] Banaszek K and Wódkiewicz K 1996 Phys. Rev. Lett. 76 4344; Wallentowitz S and Vogel W 1996 Phys. Rev. A $5 \mathbf{5 3} 4528$.

[34] Lutterbach L G and Davidovich L 1997 Phys. Rev. Lett. 78 2547; Nogues G, Rauschenbeutel A, Osnaghi S, Bertet P, Brune M, Raimond J M, Haroche S, Lutterbach L G and Davidovich L 2000 Phys. Rev. A 62054101.

[35] El-Orany F A A 2002 Phys. Rev. A 65043814.

[36] Leoński W and Miranowicz A 2004 Quant. Semiclass. Opt. 6 S37.

[37] Bajer J, Miranowicz A and Andrzejewski M 2004 Quant. Semiclass. Opt. 6387. 